\title{
Prognosis and Evolution of Lassa Virus Infected Persons during the 2017 Epidemic in Togo
}

\author{
Majesté Ihou Wateba1*, Lidawou Bawé1, Sika Dossim² \\ ${ }^{1}$ Tropical and Infectious Diseases Service of CHU SO, Lomé, Togo \\ ${ }^{2}$ Microbiology Laboratory of CHU SO, Lomé, Togo \\ Email: *majeste7@yahoo.fr
}

How to cite this paper: Wateba, M.I., Bawé, L. and Dossim, S. (2018) Prognosis and Evolution of Lassa Virus Infected Persons during the 2017 Epidemic in Togo. Advances in Infectious Diseases, 8, 262-267. https://doi.org/10.4236/aid.2018.84022

Received: November 26, 2018

Accepted: December 17, 2018

Published: December 20, 2018

Copyright ( 92018 by authors and Scientific Research Publishing Inc. This work is licensed under the Creative Commons Attribution International License (CC BY 4.0).

http://creativecommons.org/licenses/by/4.0/

(c) (i) Open Access

\begin{abstract}
Introduction: Lassa viral hemorrhagic fever is common in West Africa. Almost 300,000 persons are affected each year with 5000 deaths. The mice of the genus mastomys is the wild tank. Objective: The aim of our study is to describe clinic, therapeutic and evolution of the affected patients during February-March 2017 epidemic that occurred in the north of Togo. Methodology: Our study is a record review study from patients' record, who were hospitalized from February, $1^{\text {st }}$ to March, $31^{\text {st }} 2017$ at Mango hospital. Lassa diagnosis was performed by PCR. They patients have received Ribavirin and blood transfusion when necessary. Results: We have reported 5 clinical observations of Lassa viral hemorrhagic fever. Patients came from Benin (03 cases), from Burkina-Faso (1 case), from Togo (1 case) and were 25, 34, 60, 52 years old and a premature baby of 13 days. External hemorrhage and abdominal pains were the main symptoms. Fever was observed for all the cases. Complications were marked by hemorrhages and shocks. Only 3 patients had benefitted of Antiviral therapy with Ribavirin. The other 2 patients did not benefit from the treatment because the diagnosis of Lassa fever was done the day they dead before the treatment started. Lethality was $80 \%$ (4 cases) with a highly secured burial. Effective management of contacts was done. Conclusion: Diagnostic and therapeutic delays of patients are responsible of the bad prognosis of the disease.
\end{abstract}

\section{Keywords}

Fever, Hemorrhage, Lassa Virus, Mango (Togo)

\section{Introduction}

Lassa fever is a viral disease that belongs to the group of virus diseases causing hemorrhagic fevers, mainly in West Africa [1]. It is a public health emergency. 
Lassa fever may be the most popular of all viral hemorrhagic fevers, with a lethality of $36 \%$ to $67 \%$ among infected patients [2]. Each year, it is estimated from 100,000 to 300,000 , the number of people infected, and to 5000 , the number of deaths [3] [4]. The Lassa virus is an RNA virus. The wild tank is the mouse belonging to the genus mastomys. Human are infected by contact with the excreta of the mouse.

In this sturdy, we have reported confirms cases of patients infected by Lassa fever from February 1st to March 31st, 2017 in the northern Togo $550 \mathrm{~km}$ from the capital Lomé.

The aim of this study is to describe clinic, therapeutic and evolution of the affected patients during February-March 2017 epidemic that occurred in the north of Togo.

\section{Method}

It was a record review study of Lassa virus infected who were treated in Mango hospital during the Lassa fever outbreak in Togo between February and March 2017.

The diagnosis of Lassa fever was made by PCR. All patients were hospitalized and a blood cell count and liver checkup were performed. The treatment was based on the administration of ribavirin in 3 patients. Unfortunately, two patients had not been treated because they died on the day the diagnosis was made. Blood transfusion has been given if necessary. The clinic and therapeutic characteristics and the disease prognostic were studied.

\section{Results}

We have reported 5 confirmed cases Lassa virus fever during the epidemic in northern Togo in 2017.

Observation No. 1

It were a female, 25-year-old patient residing in Ouragaye in central-eastern Burkina Faso, admitted on February 23th, 2017 for a fever and external bleeding (ears, mouth, nose, anus and vagina). The symptoms evolved approximately 8 days before admission. Previously, she has been hospitalized in Burkina Faso for home miscarriage.

The clinical examination on the admission has founded: a fiver at $40^{\circ} \mathrm{C}$, a running pulse, a blood pressure at 100/50 $\mathrm{mm} \mathrm{Hg}$, tachypnea at 68 cycles/minute, a respiratory distress, blueness of the conjunctiva, sweats profuse, poor general condition, coma stage II (Glasgow Score at 6/15) and hemorrhagic syndrome.

Molecular biology or Polymerase Chain Reaction (PCR) has confirmed the diagnosis on February 25, 2017, 2 days after admission and 10 days after the onset of symptoms.

The management has consisted in: isolation, administration of ribavirin, oxygenation (6 liters/min), vascular filling (crystalloid + macromolecules), and transfusion of red blood cell $\mathrm{O}$ negative. 
Complications were bilateral hypoacusis, persisting and worsening hemorrhage, and Glasgow stage IV (Glasgow Score 4/15). The death occurred on March 03th, 2017 after 8 days of hospitalization.

Contacts of this patient have been identified: 135 identified contacts in Burkina Faso and 7 contacts in Togo. None of the people who had contact with the patient were infected.

Observation No. 2

This was a pregnant patient of 8 months of pregnancy, aged 34 years. Febrile for 5 days and living in a nearby locality at Benin close to the border with Togo. The symptomatology dates back to 5 days before admission, and was marked by genital bleeding, gingivorragia, pallor and arterial hypertension at 170/120 $\mathrm{mmHg}$. The general signs were dominated by fiver at $39^{\circ} \mathrm{C}$, pulse at 125 pulsations/minute, a tachypnea at 50 cycles/minute, severe pallor of conjunctiva and palmar-plantar. Her clinical condition required a caesarean on February 11 to save the baby in pain. His death occurred a few hours after the Caesarean and the diagnosis of Lassa fever was made after his death on February 13th.

The management was based on: oxygenation (6 litres/min), vascular filling (crystalloid + macromolecule), blood transfusion, antipyretic, antibiotic, and antimalarial.

The complications has consisted by persistent of the fever, respiratory distress and impaired consciousness (coma stage IV). The death occurred on February 11, 2017 after 05 days of hospitalization.

Two close contact persons were identified in relation to this case whose follow-ups were positive. These were observations 3 and 4 .

\section{Observation No. 3}

It was a male 13-day premature neonate born by cesarean at Papani (Benin) and admitted to Mango hospital in February 24, 2017 for fever. Her mother died after the cesarean in a fever context before has been confirmed positive for the Lassa virus.

In the admission, the clinical examination of the baby made it possible to note: hyperthermia at $39^{\circ} \mathrm{C}$, a pulse at 178 pulsations/minute, a blood pressure at $71 / 35 \mathrm{mmHg}$, a tachypnea at 68 cycles/minute with respiratory distress (Silverman score at 5/10) and anemic syndrome. The confirmation of a Lassa virus fever was made on February 26, 2017 by PCR, 2 days after admission.

The management has consisted of oxygenation, vascular filling, antibiotic and antimalarial treatment, administration of vitamin $\mathrm{K} 1$ and antiviral (Ribavirin).

The complications were anemic, bulging of the anterior fontanel and bleeding (nose, mouth, anus). The death occurred on March 09, 2018, after 13 days of hospitalization.

The contacts identified in Benin were 68, and 29 in Togo. Among them, one case (the grandmother of the newborn who replaced the mother after her death) was declared positive for the Lassa virus by PCR.

\section{Observation No. 4}

It was the contact person of the newborn (observation No. 3) who were the 
grandmother of the baby. In solitary confinement on February 24, 2017, she had presented on March 1, 2017, 5 days later, a retro-sternal and abdominal pain, odynophagia and fever. The clinical examination at admission noted: fever at $40^{\circ} \mathrm{C}$, pulse at 110 pulsations/minute, blood pressure at 139/83 $\mathrm{mm} \mathrm{Hg}$, tachypnea at 42 cycles/minute and inflammatory throat with swollen tonsils and whitish pultaceous deposits. The confirmation of Lassa virus infection was performed on 03 March 2017 by PCR.

The care consisted to the administration of antibiotic and antimalarial treatment; and the administration of Ribavirin.

The evolution was marked by bleeding (nose, ears, mouth) and left unilateral hypoacusis was the main complications. The evolution was favorable after 18 days of hospitalization with a negation of the PCR on March 12, 2017.

Three contacts were identified including 2 confirmed cases who died (observations 2 and 3)

Observation No. 5

He was a 52-year-old hypertensive patient residing in Gando (Togo). He has been admitted on March 12, 2017 for fever, intense physical asthenia, abdominal pain, hematemesis and disorders of consciousness. Symptomatology evolving for about 10 days before admission.

The clinical examination has noted: a fever at $38.9^{\circ} \mathrm{C}$, a pulse at 125 pulsations/minute, a blood pressure at $110 / 75 \mathrm{~mm} \mathrm{Hg}$, a tachypnea at 50 cycles/ minute with respiratory distress, a severe pallor of conjunctiva and palmar-plantar and a stage II coma (Glasgow Score at 8/15). The confirmation of Lassa fever was made on March 13, 2017, 11 days after the beginning of the signs.

Management was based on: oxygenation (6 1/min), vascular filling with crystalloid administration and macromolecules, blood transfusion, antipyretic administration, amoxicillin antibiotic therapy and antimalarial treatment with artesunate.

The complications were: the persistent fever, respiratory distress and impaired consciousness (stage IV coma). The death occurred on March 13, 2017, after 24 hours of hospitalization.

Twenty-five contacts were identified in relation to this case and no case was declared positive at the end of the 4 weeks of follow-up of these people.

\section{Discussion}

The onset of a viral hemorrhagic fever is slow and insidious, making diagnosis difficult at first, with fever appearing later [2]. The infection is unapparent and the diagnosis is commonly serological in endemic areas [5].

WHO studies on hemorrhagic viral fevers have shown that the first clinical signs of these conditions do not include hemorrhage [5] [6].

The etiological treatment of Lassa fever is based on Ribavirin [5], which is also recommended for the treatment of hemorrhagic fever caused by the Crimean Congo virus [7]. Treatment results on Lassa fever suggest that ribavirin is more effective when administered by intravenous in the first six days of the disease [5] 
[7]. In our study, only 3 patients were able to receive ribavirin. The other two were unable to receive ribavirin because the confirmatory diagnosis was obtained the same day they died. This poses the problem of the long consultation period because the African populations often resort to traditional medicine and only go to the hospital in case of failure of the latter. Oral administration should be limited to prophylaxis after high-risk exposure to Lassa fever and Crimean Congo hemorrhagic fever [7].

The serious side effects of short course ribavirin therapy are rare, but require strict monitoring. The most common side effect is, depending on the dose administered, mild or moderate hemolytic anemia, which rarely requires transfusion and regresses when therapy is stopped. Shivers can appear during a fast administration. Contraindications to its use are: severe anemia, coronary heart disease, kidney failure, liver failure, breast-feeding and known hypersensitivity to ribavirin. Jaundice can develop in patients with Gilbert's syndrome. Hemoglobin, hematocrit and bilirubin levels should be checked at the start of treatment and then every few days. If the patient develops significant anemia, consideration should be given to transfusion of red blood cell concentrates [7].

In the majority of cases, Lassa fever causes abortion in $80 \%$ of pregnant women, death of $95 \%$ of fetuses and a significant increase in maternal mortality [5].

The cases reported during this epidemic in Togo are essentially exports from Benin and Burkina Faso following epidemiological field investigations by a WHO team, with regard to the search for contact topics and the putting in place measures to fight the epidemic [8].

\section{Conclusion}

The outbreak of Lassa fever was deadly in Togo. Four out of five patients died despite the administration of ribavirin and the free management of patients. The too long delay of consultation by patients was observed in the majority of patients and would be the main cause explaining the high mortality rate. This proves the importance of prevention through the eradication of virus reservoirs and the sensitization of rural populations to seek priority consultation in hospitals in case of illness.

\section{Conflicts of Interest}

The authors declare no conflicts of interest regarding the publication of this paper.

\section{References}

[1] Saltzmann, S. (1978) The Lassafever. Missionary Edition Groups, Strasbourg, New York, p. 46.

[2] Simpson, D. (1979) Human Haemorrhagic Fever Virus. WHO Newsletter, 57, 19-32.

[3] Geibert, T., et al. (2005) Development of a New Vaccine for the Prevention of Lassa Fever. PLoS Medicine, 2, e183. https://doi.org/10.1371/journal.pmed.0020183 
[4] Surveillance of the Haemorrhagic Fever Virus and Research of Contact; Newsletter of the Ministry of Health and Population, Togo, June 2016, p. 22.

[5] Tchatcher, R. (2014) Clinical Cases Management of Haemorrhagic Fever Virus. IMAI-IMCI Alliance, Suisse, 14-16.

[6] Ehichioya, D.U., Asogun, D.A., Ehimuan, J., Okokhere, P.O., Pahlmann, M., Olschläger, S., Becker-Ziaja, B., Günther, S. and Omilabu, S.A. (2012) Hospital-Based Surveillance for Lassa Fever in Edo State, Nigeria, 2005-2008. Tropical Medicine \& International Health, 17, 1001-1004.

https://doi.org/10.1111/j.1365-3156.2012.03010.x

[7] WHO (2018) Clinical Cases Management of Haemorrhagic Fever Virus: Pocket Guide for the Front-Line Health Worker. Genève. World Health Organisation, Geneva, $222 \mathrm{p}$.

[8] WHO (2017) Lassa Fever, Bénin, Togo and Burkina Faso. Newsletter on Epidemic Outbreaks: World Health Organisation, Geneva. 